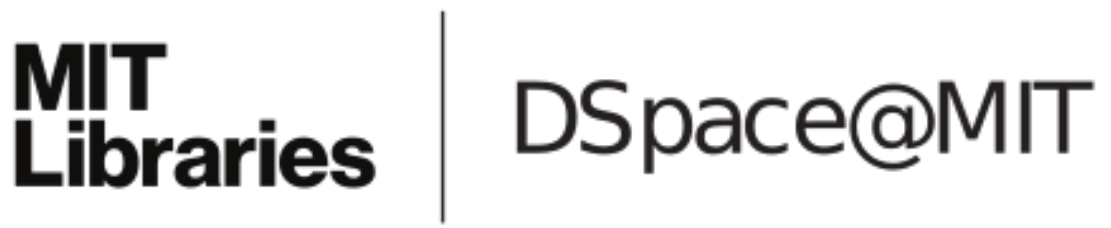

\author{
MIT Open Access Articles
}

\begin{abstract}
Modeling injected interstitial effects on void swelling in self-ion irradiation experiments
\end{abstract}

The MIT Faculty has made this article openly available. Please share how this access benefits you. Your story matters.

Citation: Short, M.P. et al. "Modeling Injected Interstitial Effects on Void Swelling in SelfIon Irradiation Experiments." Journal of Nuclear Materials 471 (April 2016): 200-207 @ 2015 Elsevier B.V.

As Published: http://dx.doi.org/10.1016/j.jnucmat.2015.10.002

Publisher: Elsevier

Persistent URL: http://hdl.handle.net/1721.1/114547

Version: Original manuscript: author's manuscript prior to formal peer review

Terms of use: Creative Commons Attribution-Noncommercial-Share Alike 


\title{
Modeling Injected Interstitial Effects on Void Swelling in Self-Ion Irradiation Experiments
}

\author{
M. P. Short; D. R. Gaston‡ M. Jin ${ }^{\S}$ L. Shao, F. A. Garner»
}

September 30, 2015

\begin{abstract}
Heavy ion irradiations at high dose rates are often used to simulate slow and expensive neutron irradiation experiments. However, many differences in the resultant modes of damage arise due to unique aspects of heavy ion irradiation. One such difference was recently shown in pure iron to manifest itself as a double peak in void swelling, with both peaks located away from the region of peak displacement damage (DPA). In other cases involving a variety of ferritic alloys there is often only a single peak in swelling vs. depth that is located very near the ion-incident surface. We show that these behaviors arise due to a combination of two separate effects: 1) suppression of void swelling due to injected interstitials, and 2) preferential sinking of interstitials to the ion-incident surface, which are very sensitive to the irradiation temperature and atomic displacement rate. Care should therefore be used in collection and interpreting of data from the depth range outside the Bragg peak of ion irradiation experiments, as it is shown to be more complex than previously envisioned.
\end{abstract}

\section{Introduction and Back- ground}

Charged particle irradiation is frequently used to simulate the effect of neutron irradiation on microstructural evolution of reactor structural alloys, but is generally conducted at much higher displacement rates in order to compress the time required to reach very high DPA levels needed to observe phenomena such

\footnotetext{
* Dept. of Nuclear Science and Engineering, Massachusetts Institute of Technology

†Corresponding Author: Tel: +1-617-347-7763, Email: hereiam@mit.edu

$\ddagger$ Idaho National Laboratory

$\S$ Dept. of Nuclear Science and Engineering, Massachusetts Institute of Technology

ๆDept. of Nuclear Engineering, Texas A\&M University

" Radiation Effects Consulting, LLC
}

as neutron-induced void swelling and embrittlement [1]. When optimized to maximize simulation of void swelling, there is not a one-to-one correspondence between microstructures observed under neutron and ion irradiation.

The primary reasons for this lack of correspondence are various consequences of the increased DPA rate, very short ranges of the bombarding ion, consequences of strong gradients in DPA rate along the ion range and local defect imbalances arising from the bombarding ion $[1,2,3,4,5,6,7,8,9,10,11]$. Additionally, there are second-order effects such as ion-induced sputtering $[1,12]$ and swelling-induced compressive states in the thin irradiated film that produce a one-dimensional (not three-dimensional as in the neutron irradiation case) flow of material toward the ion-incident surface [13, 14]. All of these processes can be described as "neutron-atypical" effects.

Swelling of both neutron and ion irradiated metals has often been shown to exhibit an incubation period before the onset of steady-state swelling. This incubation period is strongly dependent on DPA rate even in the absence of microchemical evolution $[1,3,15,16]$.

In many alloys the onset of swelling requires that a microchemical evolution involving segregation and precipitation first occur, but the rate constants involved in the point defect and precipitation sequences are usually rather different, producing different incubation behavior at different DPA rates. The very short ion ranges not only allow a strong influence of the specimen surface on defect loss and dislocation loss that are atypical of neutron irradiation, but strong gradients in DPA rate along the ion range provide an internal rate effect on defect production [4], and also provide a driving force for elemental segregation along the ion path [17], thereby influencing the microchemical evolution that often precedes the onset of swelling.

Defect imbalances arising during ion irradiation arise from forward scattering involved with ion-atom 
collisions and the spatial distribution of injected ions, the latter having a very strong effect on swelling that is designated as the injected interstitial effect $[6,7,8,9,10,11]$. Garner et al. review the result of experiments conducted on pure iron and various ferritic-martensitic alloys showing that the net effect of the various neutron-atypical aspects is to produce a depth dependence of void swelling that often bears no resemblance to the depth distribution of the atomic displacement rate [18]. Under some irradiation conditions swelling only occurs near the specimen surface, and under other conditions the swelling vs. depth is more complex, sometimes producing two peaks, neither corresponding to the position of maximum displacement rate [10].

Additionally, ion beams are often rastered to provide more spatially-uniform damage fields while introducing non-uniform temporal distributions compared to neutron irradiation. This latter effect is one of the more important neutron-atypical processes that strongly affect swelling. Since rastering and injected interstitials are both suppressive and synergistic, especially on void nucleation, their interaction must be recognized and factored into data analysis. Two recent papers demonstrate the strong influence of rastering to suppress void swelling [19, 20].

If we could ignore all of these atypical influences and examine an alloy that swells easily without much of an incubation period, one might assume that the swelling vs. depth profile should mirror the dose rate vs. depth profile, but this would be an incorrect assumption. In general, alloys of even simple composition such as solute-free Fe-Cr-Ni ternaries have been observed to exhibit a large range of swelling vs. depth profiles, frequently with no resemblance to the DPA vs. depth profile $[11,21]$.

In order to minimize these influences, the ion irradiation community has learned to conduct irradiations on simple and complex alloys by not rastering the beam and by choosing a narrow volume-slice at an examination depth that minimizes the effects not only of the ion-incident surface but especially the surprisingly strong injected interstitial effect $[7,10]$. However, the fullest utilization of the ion irradiation technique would require an understanding of the evolution of swelling vs. depth over the entire ion range, allowing extraction of data over a much larger portion of the ion range. With so many neutron-atypical processes operating simultaneously during ion irradiation, a full understanding requires that a "peel the onion" approach be used, starting with the simplest metals and alloys, moving to more complex alloys, isolating and studying each neutron-atypical process at each level of compositional and microstructural complexity.

Our approach to date has been to examine these neutron-atypical effects first in annealed pure iron and cold-worked pure iron, focusing initially on the rastering effect and the defect imbalance issues without the added complexity arising from microchemical evolution, and then focusing later on the same processes occurring in Fe-Cr binary alloys and then solute-bearing Fe-Cr alloys.

In the first of these studies we focused on void swelling of annealed pure iron in a beam-rastered irradiation, showing that the injected interstitial effect indeed suppresses swelling [10]. In the second of these studies it was shown that rastering suppresses swelling compared to non-rastered fixed beams [19]. Figure 1 of Gigax's study (here reproduced in Figure 5c) shows the two most salient features are the very strong impact of the injected interstitial effect to suppress, to very high doses, the void swelling throughout the injected ion range, and the appearance of a double-peak swelling distribution in the region in front of injected ion range. A similar observation was made in self-ion irradiations on pure nickel many years earlier [8, 9]. Occasionally, double peaks are observed in other alloys, but the possibility cannot be discounted that compositional segregation along the range might be contributing to this observation.

While the injected interstitial effect was not unexpected, the appearance of a double peak in the forerange of iron and nickel was rather surprising, with the question entertained whether it might be an experimental artifact or a real characteristic of ion irradiation. In this paper we model the time-dependent development of depth-dependent void swelling in annealed pure iron as a function of irradiation temperature and accumulated dose produced during nonrastered irradiation with $3.5 \mathrm{MeV}$ Fe ions. It is found that both the double peak and the injected interstitial suppression effects are consequences of the defect imbalance effect, reproducing the observed behavior. This model can then be used to forecast swelling behavior in pure iron and possibly more complex ironbase alloys for other temperatures, DPA rates, ion energies, rastering frequencies, etc. In particular, the double-peak is predicted to only manifest itself within a narrow window $(\sim 30 \mathrm{~K})$ of temperature, which may explain some of its previous elusiveness.

\section{Methodology}

A fully coupled implementation of the point defect kinetics equations in the form of Equations 10 and 11 in [22] was implemented in MOOSE [23], the Mul- 
tiphysics Object Oriented Simulation Environment, a finite element method framework. By fully coupled, we refer to the definition of all equations and material properties as functions of all possible independent variables (defect concentrations, temperature, ion beam current) rather than using any numerically convenient assumptions of constant or simplified material properties. Only the initial stages of point defect creation and subsequent void nucleation rates at zero time were studied, further stabilization and growth of voids typical of cluster dynamics simulations was not considered in this study.

The reader should therefore note what this study is and what it is not. It is a simple demonstration that accounting for a tiny fraction of injected interstitials greatly changes the qualitative form of the void swelling distribution. This study is not meant to be quantitative in any way, nor does it predict true, time-dependent microstructure evolution. Other recent studies using normal rate theory (NRT) and the production bias model (PBM) [22], which accounts for the creation and evolution of self-interstitial atom (SIA) clusters, treat radiation-induced microstructural evolution in a more quantitative manner.

\subsection{Equational Framework}

The point defect kinetics equations were implemented as follows:

$$
\begin{aligned}
& \frac{\partial C_{v}}{\partial t}=f_{\text {survive }} K_{O} \\
& -K_{\perp, v} \rho_{\perp} C_{v}-K_{i v} C_{v} C_{i}+D_{v} \nabla^{2} C_{v} \\
& \frac{\partial C_{i}}{\partial t}=\left[f_{\text {survive }}\left(1-f_{i-\text { cluster }}\right) K_{O}+K_{I I}\right] \\
& -K_{\perp, i} \rho_{\perp} C_{i}-K_{i v} C_{i} C_{v}+D_{i} \nabla^{2} C_{i}
\end{aligned}
$$

where $C_{v}$ and $C_{i}$ are the spatially-dependent concentrations of vacancies and interstitials in $\left[\frac{\#}{\mathrm{~nm}^{3}}\right]$, $f_{\text {survive }}$ is the intracascade point defect survival fraction (the true fraction of remaining point defects after the damage cascade quenches \& anneals), $K_{0}$ is the point defect source term from binary collisions in $\left[\frac{\#}{\mathrm{~nm}^{3}-\mathrm{s}}\right], K_{I I}$ is the injected interstitial source term in $\left[\frac{\#}{\mathrm{~nm}^{3}-\mathrm{s}}\right], K_{\perp}$ is the dislocation sink rate constant in $\left[\frac{1}{s}\right], K_{i v}$ is the vacancy/interstitial recombination rate constant in $\left[\frac{n m^{3}}{\#-s}\right]$, and $D_{v} \& D_{i}$ are the diffusivities of vacancies and interstitials in $\left[\frac{\mathrm{nm}^{2}}{\mathrm{~s}}\right]$. All

\begin{tabular}{|c|c|c|}
\hline SRIM Parameter & Value & Unit \\
\hline \hline Ion Type & $\mathrm{Fe}^{++}$ & - \\
\hline Target & Pure Fe & - \\
\hline Ion Energy & 3.5 & $\mathrm{MeV}$ \\
\hline Number of Ions & 500,000 & $\#$ \\
\hline Target Density & 7.85 & $\frac{\mathrm{g}}{\mathrm{cm}^{3}}$ \\
\hline Target Thickness & 3 & $\mu \mathrm{m}$ \\
\hline
\end{tabular}

Table 1: SRIM simulation parameters used to generate the point defect source term in this study

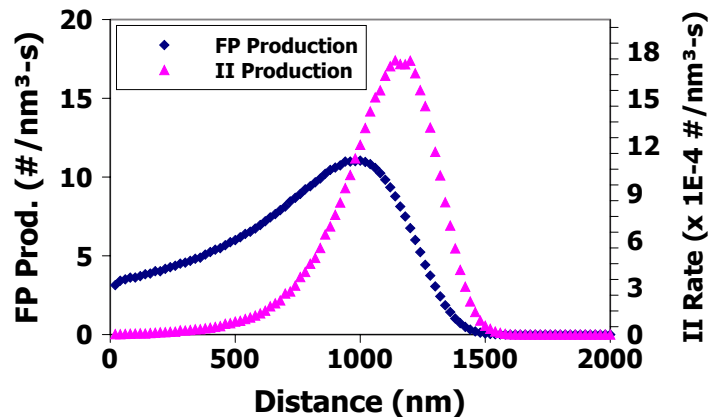

Figure 1: Frenkel pair (FP) and injected interstitial (II) production rates, as calculated by SRIM. Note the peak ballistic damage rate at $1000 \mathrm{~nm}$.

rate constants, diffusivities, and point defect concentrations are spatially dependent in one dimension on local state variables and defect concentrations. Here, an aspect of the PBM was used to assume that a fraction of interstitials immediately form highly mobile SIA clusters within each damage cascade [22], which diffuse so quickly in one dimension that they are eliminated from localized recombination and sinking. The SIA cluster concentration is therefore not tracked in this model.

The term $\mathrm{K}_{0}$ was calculated using the SRIM-2010 code [24], with simulation parameters as shown in Table 1. These parameters were chosen to match those used in the experiments of Shao et al. [10]. SRIM was run in "quick calculation" mode using the KinchinPease model, to avoid recently discovered anomalies in its full-cascade model [25]. Also, because the random number seed in SRIM does not change automatically, 50 separate simulations each containing 10,000 flown ions were averaged using a script ${ }^{1}$ to obtain smooth profiles of both the DPA rate and the injected interstitial rate as a function of distance from the free surface.

The VACANCY.TXT and RANGE.TXT files from SRIM (shown in Figure 1), which give $\frac{\text { Vacancies }}{\AA \text {-ion }}$

\footnotetext{
${ }^{1}$ All scripts, input files, and output files used in this study are permanently posted on our Github repository: [26]
} 
and $\frac{\text { injectedions }}{\mathrm{cm}}$, respectively, were converted to data files giving defect production and interstitial injection rates. The ballistic Frenkel pair (FP) production rate was multiplied by the experimentally measured beam current $(200 \mathrm{nA})$ and dividing by the beam area $(1 \mathrm{~mm}$ in diameter) in terms of the ionic flux:

$$
\begin{aligned}
& K_{0_{F P s}}(x)\left[\frac{V a c .}{n m^{3}-s e c}\right] \\
& =\left(K_{P K A s}+K_{\text {Recoils }}\right)_{S R I M}\left[\frac{\text { Vac. }}{\AA-i o n}\right] \\
& * \frac{10 \AA}{1 n m} * \frac{1 F e^{+2} i o n}{2 e^{-}} * 200 n A * \frac{1 \frac{e^{-}}{s e c}}{1.6 \cdot 10^{-10} n A} \\
& * \frac{1}{\pi\left(5 \cdot 10^{5} n m\right)^{2}}=7.96\left(K_{P K A s}+K_{\text {Recoils }}\right)_{S R I M}
\end{aligned}
$$

Simultaneously, the spatially dependent ion range distribution from SRIM was converted to total injected interstitials (II) per nm, by also multiplying by the beam current density:

$$
\begin{aligned}
& K_{0_{I I}}(x)\left[\frac{I I}{n m^{3}-s e c}\right]=R A N G E_{S R I M}\left[\frac{\frac{i o n s}{c m^{3}}}{\frac{i o n s}{c m^{2}}}\right] * \frac{1 \mathrm{~cm}}{10^{7} \mathrm{~nm}} \\
& \quad * \frac{1 F e^{+2} i o n}{2 e^{-}} * 200 n A * \frac{1 \frac{e^{-}}{\mathrm{sec}}}{1.6 \cdot 10^{-10} n A} \\
& * \frac{1}{\pi\left(5 \cdot 10^{5} \mathrm{~nm}\right)^{2}}=R A N G E_{S R I M}(x) *\left(7.96 \cdot 10^{-8}\right)
\end{aligned}
$$

Next, the dislocation sink rate constant was calculated by first finding half the average distance between dislocations $\left(\frac{1}{2} d_{\perp}\right)$ based on the dislocation density $\left(\rho_{\perp}\right)$ :

$$
\frac{1}{2} d_{\perp}=\frac{1}{\sqrt{\pi \rho_{\perp}}}
$$

and then using it to calculate the dislocation sink rate constant:

$$
K_{\perp,(v, i)}=\frac{2 \pi D_{(v, i)}}{\ln \left(\frac{\frac{1}{2} d_{\perp}}{r_{\perp(v, i)}}\right)}
$$

where $r_{\perp}$ is the core size of the dislocation for each type of defect, or the approximate radius of capture should a particular defect stray within this radius. The recombination rate constant was calculated as follows:

$$
K_{i v}=\frac{z_{i v} V_{F e}\left(D_{v}+D_{i}\right)}{a_{F e}^{2}}
$$

where $a_{F e}$ is the temperature-dependent lattice parameter of iron, $V_{F e}$ is the temperaturedependent atomic volume of iron, and $z_{i v} \sim 500$ [1]. Temperature-dependent diffusivities were calculated using an Arrhenius relation:

$$
D_{(v, i)}=D_{0} e^{\left(\frac{-E_{A}(v, i)}{k_{B} T}\right)}
$$

where $D_{0}$ is a diffusivity prefactor, $\mathrm{E}_{\mathrm{A}_{(\mathrm{v}, \mathrm{i})}}$ are the activation energies for point defect migration in $\mathrm{eV}, k_{B}$ is the Boltzmann constant $\left(8.62 \cdot 10^{-5} \frac{\mathrm{eV}}{\mathrm{K}}\right)$, and $T$ is the temperature in Kelvin. The atomic volume of iron $\left(V_{F e}\right)$ was found from molecular dynamics simulations from [27]:

$V_{F e}\left[n m^{3}\right]=1.93559 \cdot 10^{-10} T^{2}+2.68634 \cdot 10^{-7} T+0.0116954$

The thermal vacancy concentration $C_{v}^{*}$ as a function of temperature was found using molecular dynamics simulations from [27]:

$$
C_{V}^{*}\left[\frac{\#}{n m^{3}}\right]=N_{F e} \cdot 10^{[-44.5997+5.73698 \cdot \ln (T-376.952)]}
$$

where $N_{F e}$ is the atomic number density of iron, given by the following formula:

$$
N_{F e}\left[\frac{\#}{n m^{3}}\right]=\frac{\rho_{F e} \cdot N_{A}}{\left(\frac{10^{21} \mathrm{~nm}^{3}}{1 \mathrm{~cm}}\right) \cdot M M_{F e}}
$$

where $\rho_{F e}$ is the density of pure iron $\left(7.85 \frac{\mathrm{g}}{\mathrm{cm}^{3}}\right), N_{A}$ is Avogadro's number $\left(6.02 \cdot 10^{23}\right)$, and $M M_{F e}$ is the molar mass of natural iron $\left(55.865 \frac{\mathrm{g}}{\mathrm{mol}}\right)$. Table 2 summarizes material property constants and parameters assumed in this study. The migration energy of vacancies in $99.999 \%$ iron was chosen to be higher than the $0.66 \mathrm{eV}$ value for atomically pure iron [29] found by molecular dynamics or $0.55 \mathrm{eV}$ found experimentally [33], to account for realistic impurities and defect-solute binding. It was also chosen to be lower than vacancy migration energies for other BCC steels, such as the Russian alloys ChS-68 (1.08 eV) or EK-164 $(0.98 \mathrm{eV})$ [34]. The actual value is known to vary between $0.75-1.4 \mathrm{eV}$ for carbon-doped $\alpha$-Fe [35], as the binding energy of vacancy-carbon complexes at Stage III temperatures has been measured at $1.1 \mathrm{eV}$. This was the only fitting parameter in this study, and was tuned to $0.86 \mathrm{eV}$ to induce double peak formation. The sensitivity of the results to this parameter are discussed in later sections of this manuscript.

To obtain the zero-time void nucleation rate behavior, the vacancy supersaturation is first calculated as 


\begin{tabular}{|c|c|c|c|}
\hline Property & Value & Unit & Source \\
\hline \hline $\mathrm{T}$ & 450 & ${ }^{\circ} \mathrm{C}$ & Expt. \\
\hline Beam Current & 200 & $\mathrm{nA}$ & Expt. \\
\hline Beam Area & 1 & $\mathrm{~mm}^{2}$ & Expt. \\
\hline Peak DPA Rate & $4.6 \cdot 10^{-3}$ & $\frac{D P A}{s e c}$ & Expt. \\
\hline $\mathrm{D}_{0_{\mathrm{v}}}$ & $8.016 \cdot 10^{11}$ & $\frac{n m^{2}}{s}$ & {$[28]$} \\
\hline $\mathrm{D}_{0_{\mathrm{i}}}$ & $2.09 \cdot 10^{11}$ & $\frac{n m^{2}}{s}$ & {$[29]$} \\
\hline $\mathrm{E}_{\mathrm{m}_{\mathrm{v}}}$ & 0.86 & $\mathrm{eV}$ & See Text \\
\hline $\mathrm{E}_{\mathrm{m}_{\mathrm{i}}}$ & 0.17 & $\mathrm{eV}$ & {$[29]$} \\
\hline$r_{\perp_{v}}$ & 1.2 & $\mathrm{~nm}$ & {$[30]$} \\
\hline$r_{\perp_{i}}$ & 3.6 & $\mathrm{~nm}$ & {$[30]$} \\
\hline$\rho_{\perp}$ & $10^{-5}$ & $\frac{\#}{n m^{2}}$ & {$[31]$} \\
\hline $\mathrm{a}_{\mathrm{Fe}}$ & 0.286 & $\mathrm{~nm}$ & at $450^{\circ} \mathrm{C}$ \\
\hline$f_{\text {survive }}$ & 25 & $\%$ & {$[32]$} \\
\hline$f_{i-\text { cluster }}$ & 30 & $\%$ & {$[22]$} \\
\hline
\end{tabular}

Table 2: Material property parameters used in this study

follows:

$$
S_{v}=\frac{D_{v} C_{v}-D_{i} C_{i}}{D_{v} C_{v}^{*}}
$$

This study is not a quantification of the true void nucleation rate, but rather an estimation of relative effects. Therefore the general shape of a post-processed supersaturation vs. void nucleation rate curve derived from [36], which is fully summarized in [37]. It is worth noting here that while the void nucleation rate at very low dose and the void swelling rates are not directly comparable, their qualitative shapes are expected to be similar. It is well known that voids and void nuclei constitute additional point defect sinks with their own biases, more appropriately treated using Master equation [22] or cluster dynamics [38] approaches.

This underscores the point that the quantity of interest in this study is the spatially dependent vacancy supersaturation, of which the zero-time void nucleation rate is a very sensitive function. This function does not vary considerably in shape over a very wide temperature range of $200 \mathrm{~K}[36,37]$. Therefore, the same void nucleation rate curve shape is used for different conditions and materials to illustrate the strong effect of the more subtle variations in vacancy supersaturation. The curve used was for the case of $\mathrm{Ni}$ at $427^{\circ} \mathrm{C}$, with an interstitial/vacancy arrival ratio of $\frac{\beta_{i}}{\beta_{v}}=0.9$. The equation is as follows:

$$
\log \left(J_{V}\right)=5.41547 \log \left(S_{v}\right)-14.6586
$$

where $J_{V}$ is the void nucleation rate in $\left[\frac{\#}{\mathrm{~nm}^{3}-\mathrm{s}}\right]$.

\subsection{Finite Element Formulation}

A one-dimensional, time-dependent finite element simulation tool, centered around Equations 1-11, was developed using MOOSE [23]. An initial mesh size of $2 \mu \mathrm{m}$ consisting of 200 elements was chosen, as the ultimate range of $3.5 \mathrm{MeV}$ iron ions in iron was found to be $1.5 \mu \mathrm{m}$, and the SRIM data for damage production and ion range were binned into 100 spatial nodes. Zero concentration Dirichlet boundary conditions were assumed at the ends of the mesh for simplicity, and because a free surface acts as a very strong sink for defects. The SolutionDT adaptive time stepping algorithm was used to accelerate simulations when appropriate. SolutionDT works by increasing the time step by a factor of 1.5 if the current time step's solution was found to deviate very little from the previous time step.

The single matrix preconditioner (SMP) was employed to assure computational efficiency, allowing one to only specify certain off-diagonal Jacobian terms of the discretized partial differential equations (PDEs) in the problem. This preconditioner was chosen to strike a compromise between solving a Jacobian-free problem, which utilizes little computational overhead before each solve but requires more iterations to converge, and fully inverting the solution matrix, which yields an exact solution after prohibitively long times. The total residuals for each variable $C_{v}$ and $C_{i}$ are derived from the weak forms of Equations 1-2, which were implemented as follows:

$$
\begin{gathered}
\left|r^{2}\right|_{C_{v}}=0=\int_{\Omega}\left[\psi_{I} \frac{\partial C_{v}}{\partial t}-\psi_{I}\left(f_{s} K_{0}\right)+\psi_{I} K_{\perp_{v}} C_{v}\right] \\
+\int_{\Omega}\left[\psi_{I} K_{i v} C_{v} C_{i}+D_{v} \nabla \psi_{I} \nabla C_{v}\right]+\int_{\Gamma}\left[D_{v} \psi_{I} \nabla C_{v} \cdot \vec{n}\right] \\
\left|r^{2}\right|_{C_{i}}=0=\int_{\Omega}\left[\psi_{I} \frac{\partial C_{i}}{\partial t}-\psi_{I}\left(\left(f_{s} K_{0}\right)+K_{I I}\right)\right] \\
+\int_{\Omega}\left[\psi_{I} K_{i v} C_{i} C_{v}+\psi_{I} K_{\perp_{i}} C_{i}+D_{i} \nabla \psi_{I} \nabla C_{i}\right]+\int_{\Gamma}\left[D_{i} \psi_{I} \nabla C_{i} \cdot \vec{n}\right]
\end{gathered}
$$

where $\psi_{I}$ is a particular test function, $\Omega$ is the domain volume, $\Gamma$ represents the boundaries of the domain $\Omega$, and $\vec{n}$ is the surface normal vector at each boundary $\Gamma$. Reference [23] contains a more thorough description of the weak form equation development in 
the MOOSE framework. The on-diagonal and offdiagonal Jacobians for Equations 14-15 were defined as follows:

$$
\begin{aligned}
& \frac{\partial C_{v}}{\partial C_{v}(I)}=-\psi_{I} \phi_{J} \frac{\partial\left[\frac{\partial C_{v}}{\partial t}\right]}{\partial C_{v}} \\
& +\psi_{I} K_{i v} C_{i} \phi_{J}+\psi_{I} K_{\perp_{v}} \phi_{J}+D_{v} \nabla \phi_{J} \nabla \psi_{I} \\
& \frac{\partial C_{i}}{\partial C_{i}(I)}=-\psi_{I} \phi_{J} \frac{\partial\left[\frac{\partial C_{i}}{\partial t}\right]}{\partial C_{i}} \\
& +\psi_{I} K_{i v} C_{v} \phi_{J}+\psi_{I} K_{\perp_{i}} \phi_{J}+D_{i} \nabla \phi_{J} \nabla \psi_{I} \\
& \frac{\partial C_{v}}{\partial C_{v}(J)}=\psi_{I} K_{i v} \phi_{J} C_{v} \\
& \frac{\partial C_{i}}{\partial C_{i}(J)}=\psi_{I} K_{i v} \phi_{J} C_{i}
\end{aligned}
$$

where $I$ is the index (node value) for the shape function $\psi_{I}$ under consideration, $J$ is an index for a particular trial function $\phi_{J}$ under consideration. An implicit Euler time step method was used, resulting in $\frac{\partial \frac{\partial u}{\partial t}}{\partial u}=\frac{1}{\Delta t}$, where $u$ is the variable under consideration. Note that the only terms that contain an offdiagonal Jacobian entry in the solution matrix are coupled terms, where two or more variables in the constitutive PDEs are found in the same term. The only terms containing a non-zero off-diagonal Jacobian entry are the defect recombination terms, as they explicitly contain both $C_{v}$ and $C_{i}$, which are the variables used in the PDEs in this framework.

\section{Results}

First, the results of intermediate calculations are given, followed by simulation results meant to match the experiments of Shao et al. [10]. Next follows predictions for varied experimental conditions. Results are given for two cases: with and without injected interstitials, the latter case removing the $K_{I I}$ term from Equations 2 and 15.

\subsection{Defect Concentrations}

Figure 2 shows a spatial plot of the excess injected interstitial fraction, or the ratio of the total interstitialto-vacancy production rate minus one. The plot ends near the end of the FP production region, as the excess injected interstitial fraction is invalid beyond this region. Figure 3 shows the quasi-steady state defect concentrations for the cases with and without injected interstitials. Even though the excess injected interstitial fraction shows a very slight imbalance between defect production rates, in the hundreds

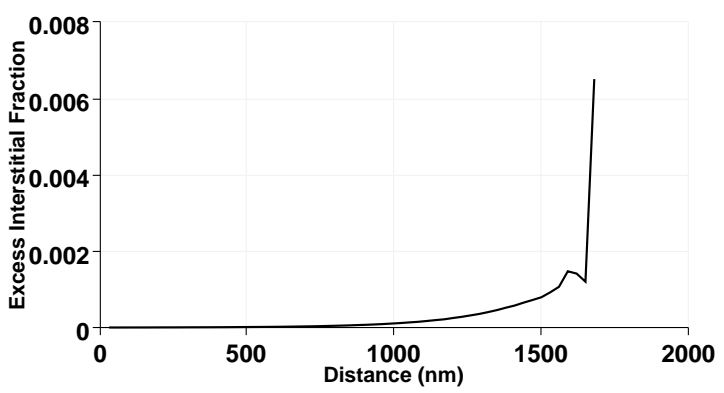

Figure 2: Spatially dependent excess interstitial fraction with injected interstitials, peaking at a value of $0.7 \%$. The bump in the curve at $1650 \mathrm{~nm}$ is an artifact of noise from the SRIM simulation.

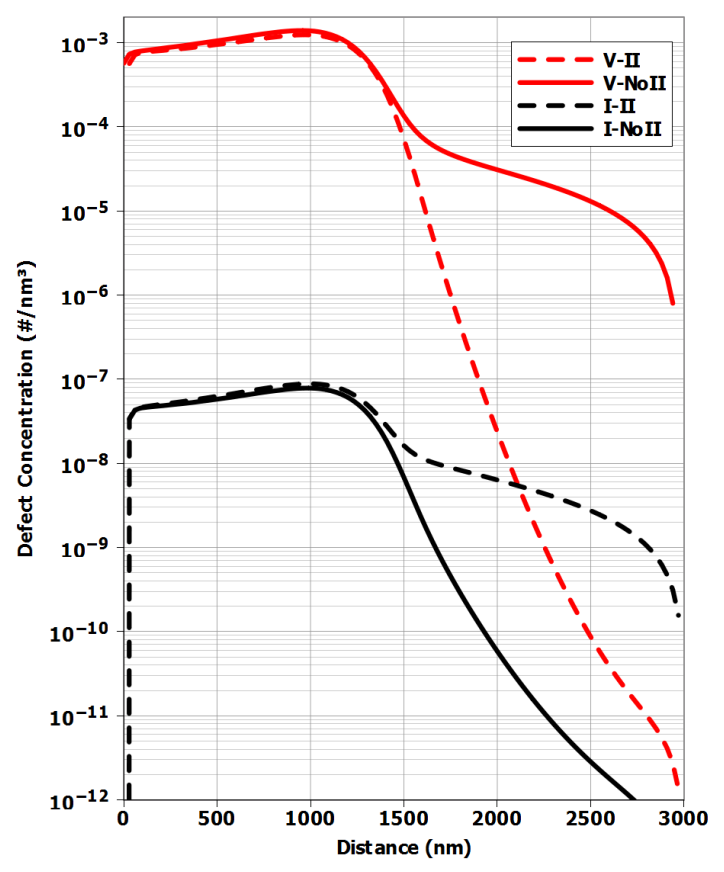

Figure 3: Spatially dependent defect concentrations, without (dashed) and with (solid) injected interstitials 


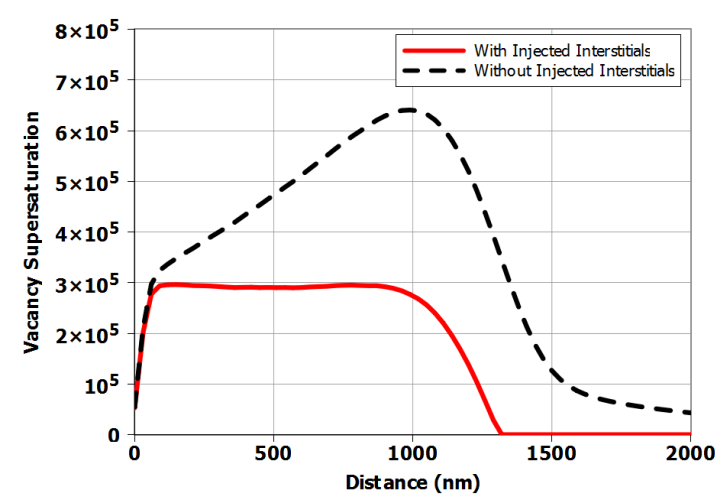

Figure 4: Vacancy supersaturation, without (black dashed) and with (solid red) injected interstitials

of ppm on average, the resulting steady-state defect concentrations differ greatly. This imbalance, especially near the Bragg peak region of the ions where SRIM calculates the most damage, causes a shift in the damage peak towards the free surface. The effect of the free surface itself, which is modeled as an undepletable sink with a zero defect concentration, is also pronounced, preferentially removing interstitials in the vicinity due to their higher mobility.

\subsection{Vacancy Supersaturation and Qualitative Void Nucleation Rate}

The resulting vacancy supersaturation curves for cases with and without injected interstitials are shown in Figure 4. Two main features are of significant interest here. Firstly, the appearance of a second peak in supersaturation, though small, is quite significant. Secondly, the pull of the free surface, combined with a $55 \%$ suppression of vacancy supersaturation at the location of peak damage by injected interstitials, "exposes" this second peak rather than inducing it.

Void nucleation rates with and without injected interstitials are shown in Figures 5a-b. The larger disparity is due to the very high sensitivity of void nucleation rate to vacancy supersaturation value, which can change by 10-15 orders of magnitude for a 10x increase in vacancy supersaturation [36]. The case with injected interstitials is presented above matching experimental data from [10], conducted under similar conditions as those simulated in this study, showing better agreement compared to the prediction without injected interstitials. However, the model does not predict enough of a swelling peak shift towards the free surface to match the experimental data. Also note how the presence of injected interstitials shifts the peak supersaturation level and void nucleation
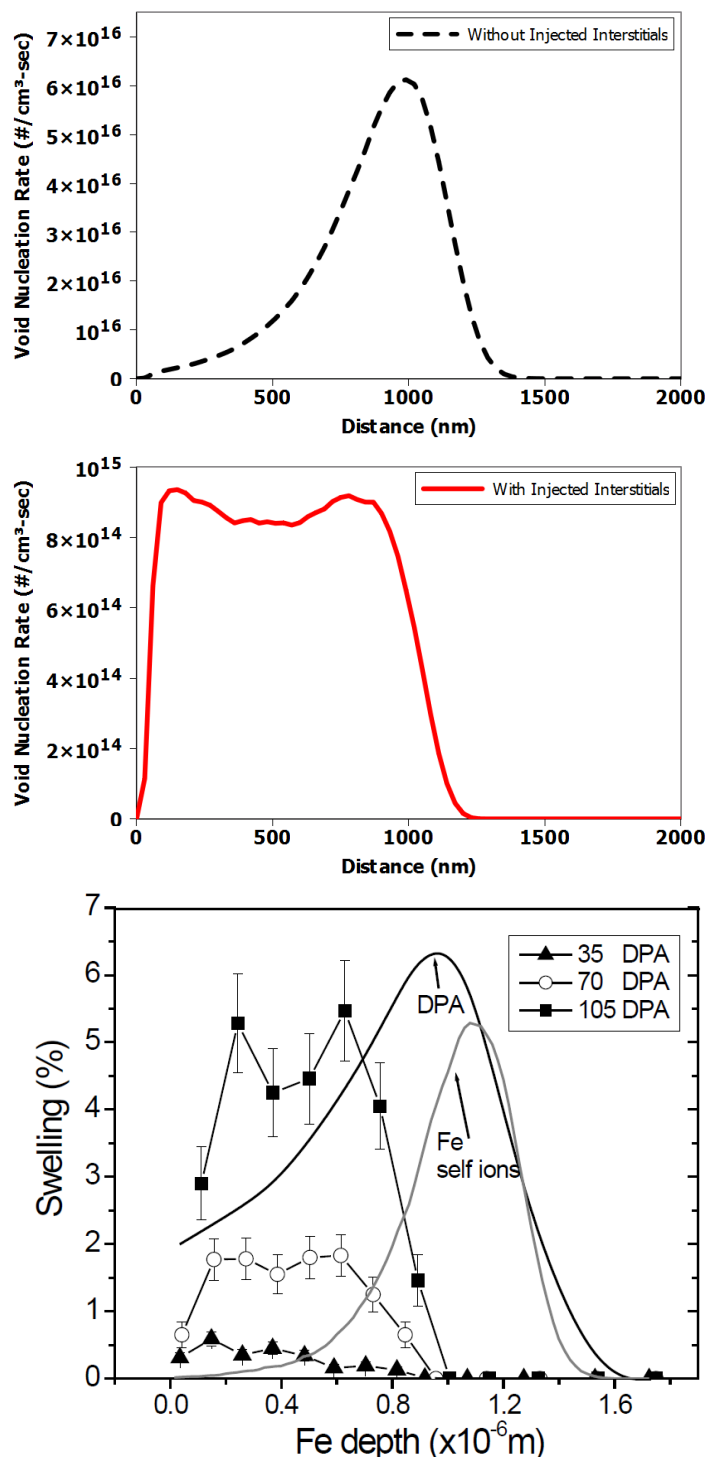

Figure 5: Void nucleation rate curve shapes, without (top) and with (middle) injected interstitials, compared to (bottom) the experimental void swelling distribution observed in $3.5 \mathrm{MeV} \mathrm{Fe}{ }^{+2}$ irradiation of pure iron [10] 


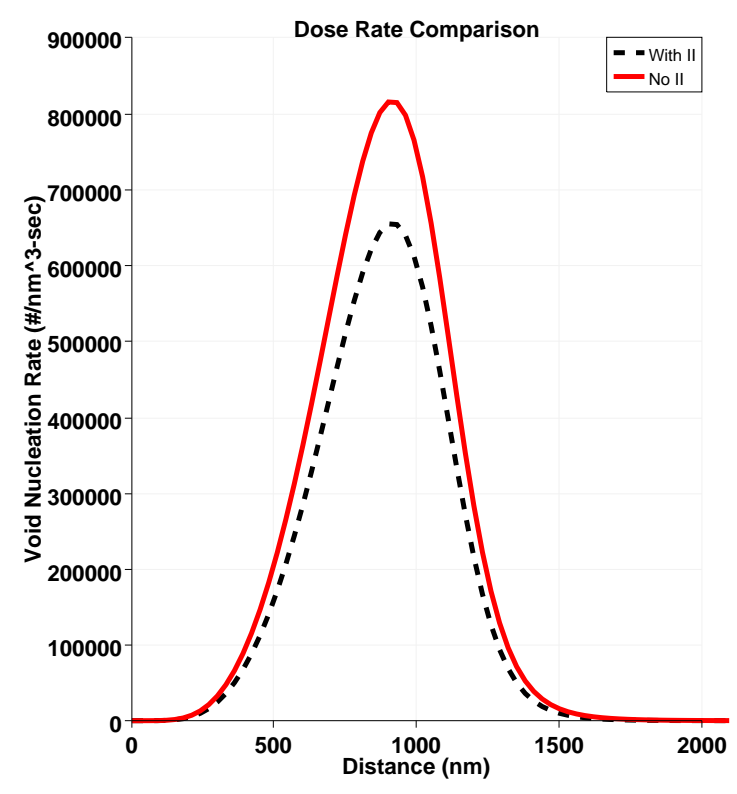

Figure 6: Void nucleation rate curves at a lower, LWR-typical peak DPA rate of $10^{-7} \frac{\mathrm{DPA}}{\mathrm{sec}}$ for $3.5 \mathrm{MeV}$ $\mathrm{Fe}^{++}$ions at $723 \mathrm{~K}$, showing no second void nucleation rate peak. Injected interstitials (dashed black line) do somewhat suppress the void nucleation rate, however.

rate location towards the free surface by $\sim 200 \mathrm{~nm}$.

\subsection{Predictions at Other Defect Pro- duction Rates, Temperatures, and Activation Energies}

A significant temperature shift has been observed $[2,3,5,39]$ and proposed $[40,41]$ by a number of researchers, whereby the temperature of peak void swelling is seen to vary between ion and neutron irradiations, with the peak temperature increasing with increasing DPA rate. The largest factor is the dose rate, which determines the defect production terms relative to invariant sink strengths, neglecting the formation of defect clusters which act as sinks themselves. Figure 6 shows plots of void nucleation rate in a typical dose rate of $10^{-7} \frac{\mathrm{DPA}}{\mathrm{sec}}$ found in a reactor. At this lower dose rate, no shifting in the peak nucleation rate position is observed. This is due to the far lower defect creation driving force compared to fixed sink strengths and densities.

Injected interstitial effect strength was also studied at different system temperatures, as summarized by Figure 7. Prevalence of the sub-surface peak becomes more apparent at lower temperatures. This is due to injected interstitials, which peak just past the location of highest damage, locally suppressing vacancy

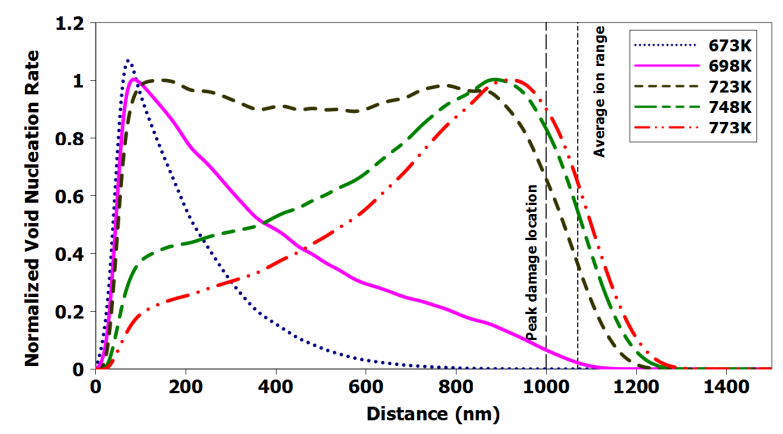

Figure 7: Normalized void nucleation rates for $3.5 \mathrm{MeV} \mathrm{Fe}{ }^{+2}$ irradiation of pure iron with injected interstitials, at temperatures ranging from $673 \mathrm{~K}$ to $773 \mathrm{~K}$

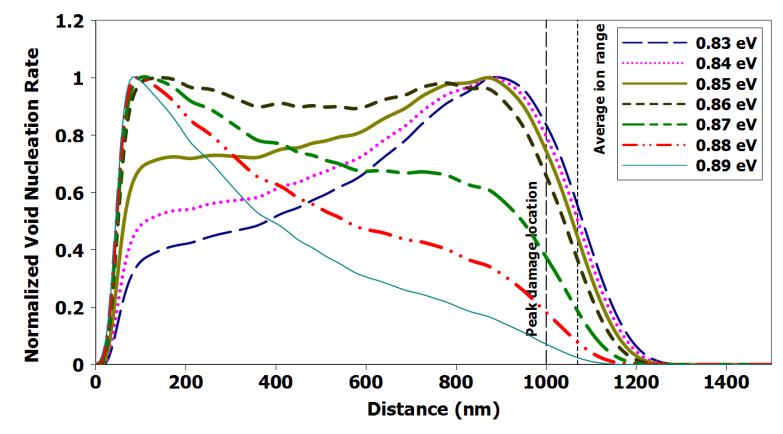

Figure 8: Normalized void nucleation rates for $3.5 \mathrm{MeV} \mathrm{Fe}{ }^{+2}$ irradiation of pure iron with injected interstitials, at vacancy activation energies ranging fro $0.83-0.89 \mathrm{eV}$

concentration as temperature decreases, and therefore decreasing local vacancy supersaturation. Locations of both sub-surface and near-peak-damage maxima shift away from the free surface with increasing temperature. For other ion energies and DPA rates, the nucleation rate profiles will be different but will preserve the features shown for $3.5 \mathrm{MeV}$.

Finally, it was found that the behavior of the void nucleation rate curve was extremely sensitive to changes in the vacancy migration energy, the only tuning variable used in this study. Figure 8 shows this dependence, in a narrow range from $0.83-0.89 \mathrm{eV}$. The fact that the results are so sensitive to this parameter implies that precise knowledge of the vacancy migration energy, which is strongly tied to ppm-level carbon impurities in iron [35], may determine whether the double-peak phenomenon is observed in a given set of experimental or simulated conditions of dose rate, temperature, and defect mobilities. 


\section{Discussion}

\subsection{Importance of the Injected Inter- stitial Effect}

The results show qualitatively that the introduction of a subtle, spatially-dependent defect imbalance may be responsible for significant changes in both magnitude and shape of spatially dependent void distributions evolving during ion irradiation. Injection of a very small fraction of injected interstitials, never more than tenths of a percent, has wide-reaching consequences. Interstitials are much more mobile, causing increased recombination at the location of peak ballistic damage, shifting the main damage peak towards the free surface by $100-200 \mathrm{~nm}$. In addition, suppression of vacancy concentration by increased recombination "exposes" the second peak near the free surface. This effect would not be seen without taking injected interstitials into account.

This indicates that the injected interstitial effect, which has been known for quite some time, must be accounted for when analyzing spatially dependent void swelling experiments employing ion beam irradiation. For example, [42] and [43] mention irradiation inducing "nearly uniform damage," in this case referring to ballistic radiation damage. A region of uniform resultant microstructural change is unlikely to form, though the higher the energy of the bombarding ion, the more uniform it may become in front of the ion stopping region. More recent studies mention taking data from a region underneath the surface, to avoid both the sub-surface and injected interstitial regions [44]. Small changes in parameters, such as temperature, dose rate, and material purity (and therefore vacancy mobility) can reveal or obscure the double peak, changing the relative magnitudes of the injected interstitial and free surface effects. Care should therefore be taken when interpreting such data from experiments. It is therefore recommended to perform a spatially-dependent simulation of defect concentrations during the irradiation experiment, to avoid misinterpretation of data from a "uniform region" which may or may not exist.

Modeling ion irradiation with and without injected interstitials at varying temperatures shows the increasing importance of the injected interstitials with decreasing vacancy mobility, as should be expected. With increasing temperature, localization of injected interstitial recombination decreases as both vacancies and interstitials become more mobile. As vacancies increase in mobility, their ability to reach the free surface from greater distances increases, shifting the subsurface peak away from the surface. In addition, as interstitials become more mobile, they move farther on average before recombining or sinking, spreading out their localized excess recombination effect.

These studies show that injected interstitial effects cannot be ignored in simulations and experiments involving ion irradiation. Depending on the temperature and the dose rate of irradiation, the resulting vacancy supersaturation, and therefore the eventual void swelling profile, can take on very different shapes. Over-generalization of ion irradiation experiment behavior should therefore be remedied, partially by taking this strong injected interstitial effect into account.

\subsection{Shortcomings of the Model}

This model is deliberately simple, neglecting the formation of defect clusters, dislocation forests, and other radiation defects which would serve as additional point defect sinks. Initial simulations using the model in this study clearly show, however, the injected interstitial effect to weaken somewhat with increased sink density, which would be expected to evolve as the material is irradiated. Therefore, a quantitative measure of the injected interstitial effect will only be possible by completely accounting for radiation-induced microstructural changes, as proposed by Golubov et al. [22]. In addition, the injected interstitial effect has been observed in far more complex materials, even though the grain boundary and incoherent precipitate sink terms are much stronger. In these alloys the observed swelling vs, depth profiles show only the near-surface peak with total suppression in the injected interstitial zone, most likely as a consequence of their very high sink density. In simple materials such as iron (this study), nickel, and even 316 stainless steel where the sink density is much lower, ion irradiation usually, but not always, leads to a double peak distribution $[9,45,46]$ depending on the ion energy, DPA rate, and irradiation temperature. In Badger's work [9] the double peak in a very pure 316 model alloy moves from a double peak to a single peak as the temperature is increased, as was observed in this study.

A full treatment of the injected interstitial effect in complex materials may be possible by implementing extra, spatially-dependent interstitial source terms found in recently developed models [47].

\section{Conclusions}

A spatially varying rate theory model for radiation defect production in ion-irradiated metals was modified to account for injected interstitials, and aug- 
mented with some elements of the production bias model (PBM). The presence of injected interstitials both suppresses and shifts the main damage peak towards the free surface, while also reducing the total vacancy supersaturation via increased recombination. This "exposes" a second peak, $200 \mathrm{~nm}$ from the free surface, which has been observed in recent experiments. Predictions were made showing differences in the strength of this effect at different temperatures and dose rates. The most important observation is that the double peak behavior shifts with increasing temperature to a single near-surface peak, in agreement with experimental observations. The double peak is predicted only to be evident within a narrow $(\sim 30 \mathrm{C})$ temperature window for self-ion irradiation of pure iron. Caution should be taken when interpreting ion irradiation data, as the ballistic damage profile may not match the resultant void swelling profile.

\section{References}

[1] G. S. Was. Fundamentals of Radiation Materials Science. Springer-Verlag, 2007.

[2] F. A. Garner and J. J. Laidler. Review of factors relevant to intercorrelation experiments. In Proceedings of Workshop on Correlation of Neutron and Charged Particle Damage, pages 177-240, Oak Ridge, TN, June 1976.

[3] F. A. Garner and et al. Summary of alloy development intercorrelation experiment. In Proceedings of Workshop on Correlation of Neutron and Charged Particle Damage, pages 147-175, Oak Ridge, TN, June 1976.

[4] F. A. Garner and G. L. Guthrie. The influence of displacement gradients on the interpretation of charged particle simulation experiments. In $R a$ diation Effects and Tritium Technology for Fusion Reactors, CONF-750989, Vol. I, pages 491518, March 1976.

[5] N. H. Packan, K. Farrell, and J. O. Stiegler. J. Nucl. Mater., 78:143-155, 1978.

[6] E. H. Lee, L. K. Mansur, and M. H. Yoo. J. Nucl. Mater., 85-86:577-581, 1979.

[7] F. A. Garner. J. Nucl. Mater., 117:177-197, 1983.

[8] D. B. Bullen, G. L. Kulcinski, and R. A. Dodd. J. Nucl. Mater., 122-123:584-589, 1984.
[9] B. Badger et al. ASTM STP 870, pages 297-316, 1985.

[10] L. Shao et al. J. Nucl. Mater., 453:176-181, 2014.

[11] R. L. Sindelar, R. A. Dodd, and G. L. Kulcinski. ASTM STP 870, pages 330-340, 1985.

[12] W. G. Johnston et al. J. Nucl. Mater., 46:273280, 1973.

[13] F. A. Garner, G. L. Wire, and E. R. Gilbert. Stress effects in ion-bombardment experiments. In Radiation Effects and Tritium Technology for Fusion Reactors, CONF-750989, pages I-474, 1976.

[14] W. G. Wolfer and F. A. Garner. J. Nucl. Mater., 85-86:583-589, 1979

[15] F. A. Garner. Comprehensive Nuclear Materials, volume 4, pages 33-95. Elsevier, 2012.

[16] F. A. Garner. Void swelling and irradiation creep in light water reactor (LWR) environments. In P. G. Tipping, editor, Understanding and Mitigating Ageing in Nuclear Power Plants, pages 308-356. Woodhouse Publishing, 2010.

[17] V. A. Pechenkin, A. D. Chernova, and F. A. Garner. Modeling of local changes in composition of alloys along the projected range under heavy ion irradiation. In Proceedings of Accelerator Applications, Bruges, Belgium, 2013.

[18] F. A. Garner et al. Use of self-ion bombardment to study void swelling in advanced radiationresistant alloys. In 17th International Conference on Environmental Degradation of Materials in Nuclear Power Systems - Water Reactors, Ontario, Canada, August 2015. (Submitted).

[19] J. Gigax et al. J. Nucl. Mater., 465:343-348, 2015.

[20] E. Getto et al. J. Nucl. Mater., 465:116-126, 2015.

[21] W. G. Johnston et al. J. Nucl. Mater., 62:167180, 1976.

[22] S. I. Golubov, A. V. Barashev, and R. E. Stoller. Comprehensive Nuclear Materials, chapter 1.13 - Radiation Damage Theory, pages 358-389. Elsevier, 2012.

[23] D. Gaston et al. Nucl. Eng. Des., 239(10):17681778,2009 
[24] J. F. Ziegler, M. D. Ziegler, and J. P. Biersack. Nucl. Inst. Methods in Phys. Res. B, 268(11-12): 1818-1823, 2010.

[25] R. E. Stoller et al. Nucl. Inst. Methods in Phys. Res. B, 310:75-80, 2013.

[26] M. P. Short et al. Github Repository, May 2015. URL http://dx.doi.org/10.5281/zenodo.31640. DOI: $10.5281 /$ zenodo. 31640 .

[27] M. I. Mendelev and Y. Mishin. Phys. Rev. B, 80:144111, 2009.

[28] V. A. Borodin and P. V. Vladimirov. J. Nucl. Mater., 362, 2007.

[29] N. Soneda and T. D. de la Rubia. Phil. Mag. A, 81, 2001.

[30] V. Shastry and T. D. de la Rubia. Trans. ASME, 121:126-128, 1999.

[31] H. Bhadeshia and R. Honeycombe. Steels: Microstructure and Properties: Microstructure and Properties. Butterworth-Heinemann, 2011. p. 23.

[32] L. Malerba. J. Nucl. Mater., 351(1-3):28-38, 2006.

[33] H. J. Wollenberger. Physical Metallurgy, chapter 18: Point Defects, pages 1621-1721. Elsevier, Amsterdam, 1996. ISBN: 978-0-444-89875-3.

[34] A. V. Kozlov et al. Russian Metallurgy (Mettaly), 2014(5):412-418, 2014.

[35] S. Takaki et al. Rad. Eff., 79(1-4):87-122, 1983.

[36] J. L. Katz and H. Wiedersich. J. Chem. Phys., 55:1414-1425, 1971.

[37] H. Wiedersich and J. L. Katz. Adv. Colloid Interface Sci., 10:33-71, 1972.

[38] T. Jourdan, G. Bencteux, and G. Adjanor. J. Nucl. Mater., 444:298-313, 2014.

[39] M. Terasawa et al. The influence of metallurgical variables on void swelling in type 316 stainless steel. In Proceedings of Radiation Effects in Breeder Reactor Structural Materials, pages 687-707, Scottsdale, AZ, USA, 1977. AIME.

[40] J. L. Straalsund. J. Nucl. Mater., 51(3):302-308, July 1974. ISSN 0022-3115.

[41] L. K. Mansur. J. Nucl. Mater., 78:156-160, 1978.
[42] T. R. Allen et al. J. ASTM Intl., 2(3), 2005.

[43] J. M. Cookson et al. J. Nucl. Mater., 202(1-2): 104, 1993.

[44] X. Wang et al. J. Nucl. Mater., 462(0):119-125, July 2015. ISSN 0022-3115.

[45] T. Aruga, Y. Katano, and K. Shiraishi. J. Nucl. Mater., 122-123:191-195, 1984.

[46] A. Bhattacharya. Ion irradiation effects on high purity bcc Fe and model $\mathrm{FeCr}$ alloys. Ph.D. thesis, Universite Paris-Sud, September 2014.

[47] A. V. Barashev, S. I. Golubov, and R. E. Stoller. A model of radiation-induced microstructural evolution. Technical report, Oak Ridge National Laboratory, 2014. ORNL/LTR-2014/487. 